\title{
Holographic Superconductors with Hořava-Lifshitz Black Holes
}

\author{
Rong-Gen Cai* and Hai-Qing Zhangt \\ Key Laboratory of Frontiers in Theoretical Physics, \\ Institute of Theoretical Physics, Chinese Academy of Sciences, \\ P.O. Box 2735, Beijing 100190, China
}

\begin{abstract}
We discuss the phase transition of planar black holes in Hořava-Lifshitz gravity by introducing a Maxwell field and a complex scalar field. We calculate the condensates of the charged operators in the dual CFTs when the mass square of the complex scalar filed is $m^{2}=-2 / L^{2}$ and $m^{2}=0$, respectively. We compute the electrical conductivity of the Hořava-Lifshitz superconductor in the probe approximation. In particular, it is found that there exists a spike in the conductivity for the case of the operator with scaling dimension one. These results are quite similar to those in the case of Schwarzschild-AdS black holes, which demonstrates that the holographic superconductivity is a robust phenomenon associated with asymptotic AdS black holes.
\end{abstract}

\section{INTRODUCTION}

The AdS/CFT correspondence [1, 2, 3] relates a weak coupling gravity theory in an antide Sitter space to a strong coupling conformal field theory in one less dimensions. Recently it has got a lot of applications in condensed matter physics [4, 5], in particular in understanding some phenomena such as superconductivity [6, 7] and superfluid [8, 9]. It turns out that a simply Einstein-Maxwell theory coupled to a charged complex scalar field could provide a holographic description of a superconductor.

The hairy black hole with a complex scalar field [10] can be explained as a charged condensation in the dual CFTs according to the AdS/CFT correspondence. The expectation value of charged operators in the dual CFTs will break U(1) symmetry; According to Weinberg [11], this produces a superconducting phase. In the paper [7], the authors considered the holographic superconductors in the Abelian-Higgs model within the probe limit, which means the backreaction of the dynamical matter field on the spacetime metric is negligible. The probe limit is justified when the charge of the complex scalar field is large. For the Einstein-Abelian-Higgs system, one has a consistent solution: a Schwarzschild-AdS black hole solution with a constant scalar field. When the black hole temperature $T$ decreases and is below a critical temperature $T_{c}$, it is found that the Schwarzschild-AdS black hole becomes unstable, and a new black hole solution with charged scalar hair is favored. It implies that a second order phase transition happens from the Schwarzschild-AdS black hole to the hairy black hole. In the field theory side, it indicates the condensation of the charged operator

\footnotetext{
*Electronic address: cairg@itp.ac.cn

${ }^{\dagger}$ Electronic address: hqzhang@itp.ac.cn
} 
comes out and a superconducting phase occurs. According to the AdS/CFT correspondence, the Maxwell field in the bulk is dual to a $U(1)$ conserved current in the boundary field theory. Therefore, considering the fluctuation of the Maxwell field, one is able to calculate the conductivity. The authors of [7] found that the behaviors of the condensation and the conductivity are qualitatively consistent with the BCS theory [12].

The backreaction effect of matter field has been studied in [13], the phase diagram and conductivity are also still consistent with the BCS theory, as expected. Extensions of the holographic superconductor to higher spin case have been done in [14, 15, 16]. The interesting holographic superconductor models have been embedded in string/M theory [17, 18, 19, 20]. More recently, the investigation of the ground state (zero-temperature limit) of holographic superconductors has revealed that there may exist some Lifshitz scaling symmetry for the bulk solutions and bulk solutions have vanishing entropy [19, 21, 22, 23].

Recently some interest arises to build the holographic superconductors in the bulk backgrounds with Lifshitz/Schrödinger scaling symmetry, according to the AdS/NCFT (nonrelativistic conformal field) correspondence [24, 25, 26]. On the other hand, more recently a

lot of attention has been focused on Hořava-Lifshitz theory [27], which is a power accounting renormalizable gravity theory. In particular, the Hořava-Lifshitz theory is a non-relativistic theory. Note that the fact that superconductivity is a phenomenon which can be described by a non-relativistic field theory. It is therefore of interest to study the holographic superconductivity in the Hořava-Lifshitz theory, partially in order to see how the phenomenon of the charged condensation is robust, and partially to see what difference will appear, compared to the case of the relativistic general relativity.

This paper is organized as follows. In Sec. П] we briefly introduce the Hořava-Lifshitz gravity including its action, planar black hole solution and associated thermodynamics. Sec. III considers the phase transition of the Hořava-Lifshitz holographic superconductors by introducing a complex scalar field and Maxwell field into the planar black hole background. The condensate of the charged operators is calculated numerically. In Sec. IV] we calculate the electrical conductivity in the probe approximation. We find that the behavior of the conductivity is qualitatively consistent with the BCS theory and a spike will appear in the conductivity if the operator is of scaling dimension one. Employing the method in [22], we try to qualitatively explain this phenomenon using the one-dimensional Schrödinger equation. We conclude our paper in Sec. V]

\section{BLACK HOLES IN HOŘAVA-LIFSHITZ GRAVITY}

In non-relativistic field theory, space and time have different scalings, which is called anisotropic scaling,

$$
x^{i} \rightarrow b x^{i}, \quad t \rightarrow b^{z} t, \quad i=1,2,3 .
$$

where $z$ is called dynamical critical exponent. In order to present the non-relativistic HořavaLifshitz gravity, it turns out convenient to employ the ADM formalism

$$
d s^{2}=-N^{2} d t^{2}+g_{i j}\left(d x^{i}+N^{i} d t\right)\left(d x^{j}+N^{j} d t\right) .
$$


where $N$ is the lapse function, $N^{i}$ is the shift vector, and $g_{i j}$ is the metric of the spacelike hypersurface. The extrinsic curvature for the hypersurface is

$$
K_{i j}=\frac{1}{2 N}\left(\dot{g_{i j}}-\nabla_{i} N_{j}-\nabla_{j} N_{i}\right),
$$

where the dot denotes the derivative with respect to time $t$, and $\nabla_{i}$ stands for the covariant derivative associated with the metric $g_{i j}$. In order for a theory to be power counting renormalizable, the critical exponent has at least $z=3$ in four spacetime dimensions. The action of the Hořava-Lifshitz theory with $z=3$ is [27]

$$
\begin{aligned}
S= & \int d t d^{3} x \sqrt{g} N\left[\frac{2}{\kappa^{2}}\left(K_{i j} K^{i j}-\tilde{\lambda} K^{2}\right)+\frac{\kappa^{2} \mu^{2}\left(\Lambda R-3 \Lambda^{2}\right)}{8(1-3 \tilde{\lambda})}+\frac{\kappa^{2} \mu^{2}(1-4 \tilde{\lambda}) R^{2}}{32(1-3 \tilde{\lambda})}\right. \\
& \left.-\frac{\kappa^{2} \mu^{2}}{8} R_{i j} R^{i j}+\frac{\kappa^{2} \mu \epsilon^{i j k}}{2 w^{2}} R_{i l} \nabla_{j} R_{k}^{l}-\frac{\kappa^{2}}{2 w^{4}} C_{i j} C^{i j}\right]
\end{aligned}
$$

where $\kappa^{2}, \tilde{\lambda}, \mu, w^{2}$ are the coupling constants and $\Lambda$ is related to the effective cosmological constant; $C_{i j}$ is the Cotten tensor, defined as

$$
C^{i j}=\epsilon^{i k l} \nabla_{k}\left(R_{l}^{j}-\frac{1}{4} R \delta_{l}^{j}\right) .
$$

In the Hořava-Lifshitz theory, the topological black hole solutions with constant scalar curvature horizon in the case of $\tilde{\lambda}=1$ are presented in [28]; the metric is of the form

$$
d s^{2}=-N_{0}^{2} f(r) d t^{2}+\frac{d r^{2}}{f(r)}+r^{2} d \Omega_{k}^{2} .
$$

where $N_{0}$ is a constant, which can be set to the unity, $d \Omega_{k}^{2}$ is a 2-dimensional Einstein space with constant scalar curvature $2 k$ (without loss of generality, $k$ can be taken 0 and \pm 1 ). The metric function $f$ is given by

$$
f(r)=k+\tilde{x}^{2}-\sqrt{c_{0} \tilde{x}} .
$$

where $\tilde{x}=\sqrt{-\Lambda} r, c_{0}$ is an integration constant and $c_{0}>0$. The mass of this topological black hole solution is

$$
M=\frac{\kappa^{2} \mu^{2} \sqrt{-\Lambda} \Omega_{k}}{16} c_{0},
$$

where $\Omega_{k}$ is the volume of the 2-dimensional Einstein space. And the Hawking temperature of the black hole is

$$
T=\frac{3 \tilde{x}_{+}^{2}-k}{8 \pi \tilde{x}_{+}} \sqrt{-\Lambda} .
$$

where $\tilde{x}_{+}$is the largest root of $f(r)=0$. 


\section{THE CONDENSATE OF CHARGED OPERATORS}

We start with the planar Hořava-Lifshitz black holes with $k=0$. In that case the metric (6) can be written as

$$
d s^{2}=-f(r) d t^{2}+\frac{d r^{2}}{f(r)}+r^{2}\left(d x^{2}+d y^{2}\right)
$$

with

$$
f=\tilde{x}^{2}-\sqrt{c_{0} \tilde{x}}
$$

One can see that in the large $r$ limit or when $c_{0}=0$, the spacetime becomes a four dimensional anti-de Sitter space, $\mathrm{AdS}_{4}$. Note that the solution (10) has very unusual asymptotic behavior, compared to the Schwarzschild-AdS black hole. Comparing with the standard $\mathrm{AdS}_{4}$ spacetime, we may set $-\Lambda=\frac{1}{L^{2}}$, where $L$ is the radius of $\mathrm{AdS}_{4}$.

The Lagrangian for the system of the Maxwell field and the complex scalar field is of the Abelian-Higgs type [7]

$$
\mathcal{L}=-\frac{1}{4} F^{\mu \nu} F_{\mu \nu}-\left|\nabla_{\mu} \psi-i q A_{\mu} \psi\right|^{2}-V(|\psi|),
$$

where $F^{\mu \nu}$ is the Maxwell field strength $F=d A$ and $\psi$ is the complex scalar field with the potential $V$. We have from (12) the equation of the scalar field

$$
-\left(\nabla_{\mu}-i q A_{\mu}\right)\left(\nabla^{\mu}-i q A^{\mu}\right) \psi+\frac{\partial V(|\psi|)}{\partial \psi^{*}}=0
$$

while the Maxwell's equation reads

$$
\nabla^{\mu} F_{\mu \nu}=i q\left(\psi^{*}\left(\nabla_{\nu}-i q A_{\nu}\right) \psi-\psi\left(\nabla_{\nu}+i q A_{\nu}\right) \psi^{*}\right)
$$

Consider the ansatz

$$
A_{\mu}=(\phi(r), 0,0,0), \quad \psi=\psi(r) .
$$

This ansatz implies that the phase factor of the complex scalar field is a constant. Thus one may take $\psi$ to be real. In the black hole background (10), the equation of scalar field (13) then reduces to

$$
\psi^{\prime \prime}+\left(\frac{f^{\prime}}{f}+\frac{2}{r}\right) \psi^{\prime}+\frac{q^{2} \phi^{2}}{f^{2}} \psi-\frac{1}{2 f} \frac{\partial V(\psi)}{\partial \psi}=0,
$$

where a prime denotes the derivative with respect to $r$, and the Maxwell's equations is simplified to

$$
\phi^{\prime \prime}+\frac{2}{r} \phi^{\prime}-\frac{2 q^{2} \psi^{2}}{f} \phi=0 .
$$

At the black hole horizon $r=r_{+}$, one must have $\phi=0$ because its norm is required finite, and the scalar field $\psi$ should also be finite there, namely, $\left.\psi\right|_{r=r_{+}}<+\infty$. 
In order to get the explicit behavior of $\phi(r)$ and $\psi(r)$ on the boundary $r \rightarrow \infty$. We assume the potential to be $V(\psi)=m^{2} \psi^{2}$ and consider two cases, one is $m^{2} L^{2}=-2$ and the other is $m^{2}=0$; both are above the Breitenlohner-Freedman bound [29]. From now on, we set the radius of $\mathrm{AdS}_{4}$ to be $L=1$. Let us first discuss the case with $m^{2}=-2$. From (16) and (17), we can easily get their behavior in the large $r$ limit,

$$
\left.\psi\right|_{r \rightarrow \infty}=\frac{\psi^{(1)}}{r}+\frac{\psi^{(2)}}{r^{2}}+\cdots,
$$

and

$$
\left.\phi\right|_{r \rightarrow \infty}=\mu-\frac{\rho}{r}+\cdots,
$$

where $\mu$ is the chemical potential and $\rho$ is the charge density on the boundary. Because the boundary is a $(2+1)$-dimensional field theory, $\mu$ is of mass dimension one and $\rho$ is of mass dimension two. From the boundary behaviors, we can read off the expectation values of operator $\mathcal{O}$ dual to the field $\psi$. From [30], we know that for $\psi$, both of these falloffs are normalizable, and in order to keep the theory stable, we should either impose

$$
\psi^{(1)}=0, \quad \text { and } \quad\left\langle\mathcal{O}_{2}\right\rangle=\sqrt{2} \psi^{(2)},
$$

or

$$
\psi^{(2)}=0, \quad \text { and } \quad\left\langle\mathcal{O}_{1}\right\rangle=\sqrt{2} \psi^{(1)} .
$$

The factor of $\sqrt{2}$ is a convenient normalization as in [7]. The index $i$ in $\psi^{(i)}$ represents the scaling dimension $\lambda_{\mathcal{O}}$ of its dual operator $\left\langle\mathcal{O}_{i}\right\rangle$, i.e., $\lambda_{\mathcal{O}_{i}}=i$.

We now find the numerical solutions of Eqs. (16) and (17) with the boundary conditions mentioned above. Because the dimension of temperature $T$ is of mass dimension one, the ratio $T^{2} / \rho$ is dimensionless. Therefore increasing $\rho$, while $T$ is fixed, is equivalent to decrease $T$ while $\rho$ is fixed. In our calculation, we find that when $\rho>\rho_{c}$, the operator condensate will appear; this means when $T<T_{c}$ there will be an operator condensate, that is to say, the superconducting phase occurs.

We plot in Fig. 1 the condensates of operators $\mathcal{O}_{1}$ and $\mathcal{O}_{2}$. They approach to fixed constants as $T \rightarrow 0$, which is qualitatively consistent with that obtained in BCS theory. In the BCS theory, the expectation values of $\left\langle\mathcal{O}_{1}\right\rangle$ and $\sqrt{\left\langle\mathcal{O}_{2}\right\rangle}$ are twice the superconducting gap. The theoretical prediction for the expectation value is $2 \times$ gap $=3.54 T_{c}$ at $T=0$ [31. . In our holographic model, however, we see from Fig. 1 that the expectation values of the two operators are higher than $3.54 T_{c}$. This may be explained that the holographic model describes a strong interacting theory than the BCS theory [5].

In the mean field theory, near $T \sim T_{c}$, the order parameter has the behavior

$$
\left\langle\mathcal{O}_{1}\right\rangle \sim\left\langle\mathcal{O}_{2}\right\rangle \sim\left(T_{c}-T\right)^{1 / 2} .
$$

In our case, we can fit the data near $T \rightarrow T_{c}$ and find

$$
\left\langle\mathcal{O}_{1}\right\rangle \approx 13.17 T_{c} \sqrt{1-T / T_{c}}, \text { when } T \rightarrow T_{c},
$$



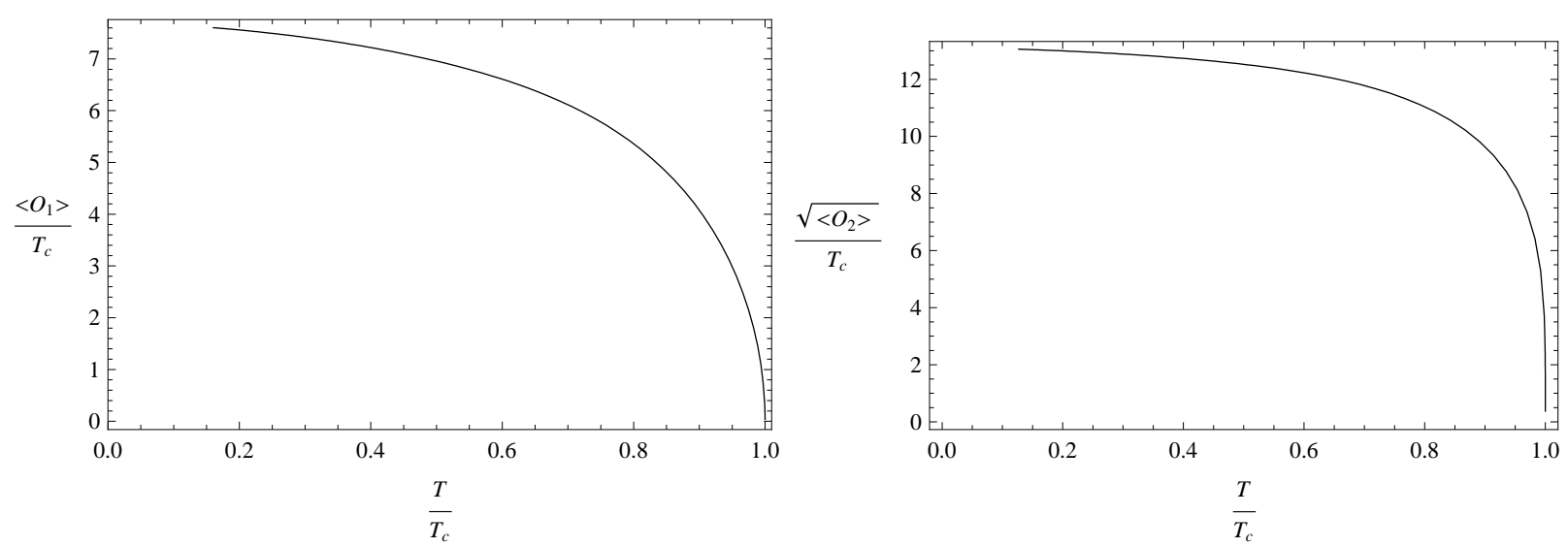

FIG. 1: The condensates of operators $\mathcal{O}_{1}$ (left) and $\mathcal{O}_{2}$ (right) versus temperature. The condensates disappear as $T \rightarrow T_{c}$.

where $T_{c} \approx 0.0406243 \sqrt{\rho}$, while

$$
\left\langle\mathcal{O}_{2}\right\rangle \approx 304.542 T_{c}^{2} \sqrt{1-T / T_{c}}, \quad \text { when } \quad T \rightarrow T_{c}
$$

where $T_{c} \approx 0.0762589 \sqrt{\rho}$. These results are qualitatively the same as the case for Schwarzschild-AdS black hole [7].

To see the condensate dependence on the mass of the scalar field, we will consider the case with $m^{2}=0$. In that case, the asymptotic behavior of scalar field near the boundary is

$$
\left.\psi\right|_{r \rightarrow \infty}=\psi^{(0)}+\frac{\psi^{(3)}}{r^{3}}+\cdots
$$

where $\psi^{(0)}$ is non-normalizable, which therefore we will not consider any more and $\psi^{(3)}$ is now proportional to the expectation value of the dual operator $\mathcal{O}_{3}$, whose scaling dimension is 3 . Near the critical temperature, we find

$$
\left\langle\mathcal{O}_{3}\right\rangle=5166.193 T_{c}^{3} \sqrt{\left(1-T / T_{c}\right)}, \quad \text { when } T \rightarrow T_{c} .
$$

where $T_{c} \approx 0.04988 \sqrt{\rho}$. To compare different behaviors of condensate for operators with different scaling dimensions, we plot the condensate of the operator $\mathcal{O}_{3}$ with the other two in Fig 2. It shows that the condensate increases when the scaling dimension $\lambda$ gets larger, which is consistent with the argument given in [32].

\section{THE ELECTRICAL CONDUCTIVITY}

In order to compute the electrical conductivity, according to the AdS/CFT dictionary, we perturb the Maxwell field and the time-space component of the black hole background, then calculate the linear response to the perturbation. In the probe approximation, the effect of the perturbation of metric can be ignored. In addition, we assume that the perturbation 


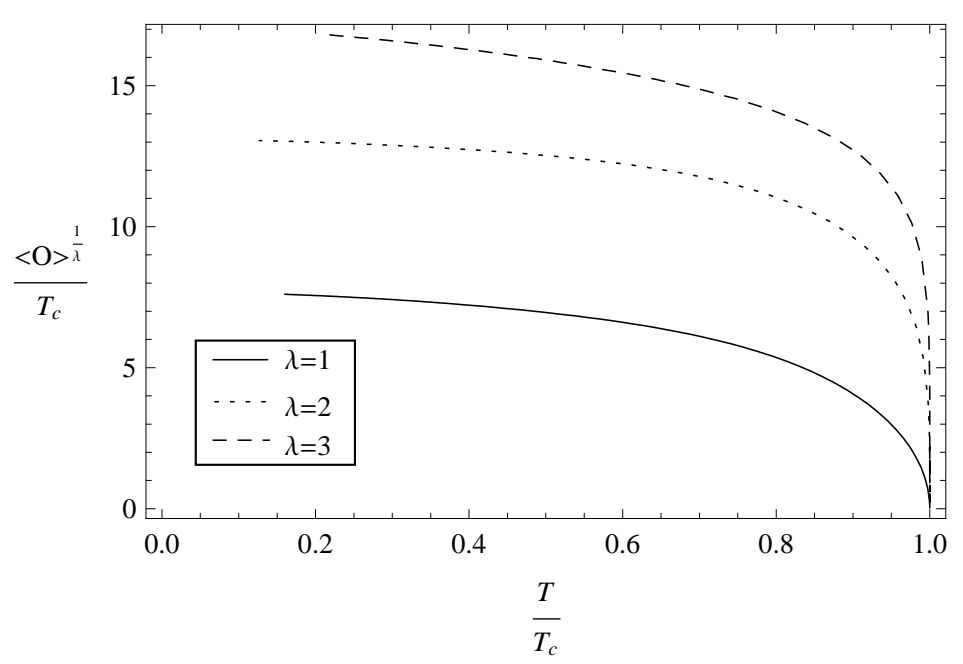

FIG. 2: The condensate of three operators with scaling dimensions, which are labelled by $\lambda$. It can be seen that the condensate increases when $\lambda$ gets larger.

of the vector potential is translational symmetric and has a time dependence as $\delta A_{x}=$ $A_{x}(r) e^{-i \omega t}$. Substitute this perturbation into Maxwell's equations (14), we have

$$
A_{x}^{\prime \prime}+\frac{f^{\prime}}{f} A_{x}^{\prime}+\left(\frac{\omega^{2}}{f^{2}}-\frac{2 q^{2} \psi^{2}}{f}\right) A_{x}=0 .
$$

At the black hole horizon, the ingoing wave condition should be employed, $A_{x} \propto f^{-i \omega / 3 r_{+}}$, while at the infinity boundary, the asymptotic behavior of $A_{x}$ is

$$
A_{x}=A_{x}^{(0)}+\frac{A_{x}^{(1)}}{r}+\cdots
$$

In AdS/CFT correspondence, it is well-known that $A_{x}^{(0)}$ corresponds to the source and $A_{x}^{(1)}$ to the expectation value for the current in the dual CFT,

$$
A_{x}=A_{x}^{(0)}, \quad\left\langle J_{x}\right\rangle=A_{x}^{(1)} .
$$

By the Ohm's law, the conductivity can be calculated as

$$
\sigma(\omega)=\frac{\left\langle J_{x}\right\rangle}{E_{x}}=-\frac{i\left\langle J_{x}\right\rangle}{\omega A_{x}}=-\frac{i A_{x}^{(1)}}{\omega A_{x}^{(0)}} .
$$

\section{A. The conductivity for the cases of $m^{2}=-2 / L^{2}$ and $m^{2}=0$}

We calculate the conductivity for the cases of $m^{2}=-2 / L^{2}$ and $m^{2}=0$. The numerical results are plotted in Fig. 3. For all plots, the real part of the conductivity approaches to $\operatorname{Re}[\sigma] \rightarrow 1$ when the frequency grows. This means the state of the dual CFT becomes a normal one when $\omega \rightarrow \infty$. The imaginary part of conductivity $\operatorname{Im}[\sigma]$ goes to infinity when the 

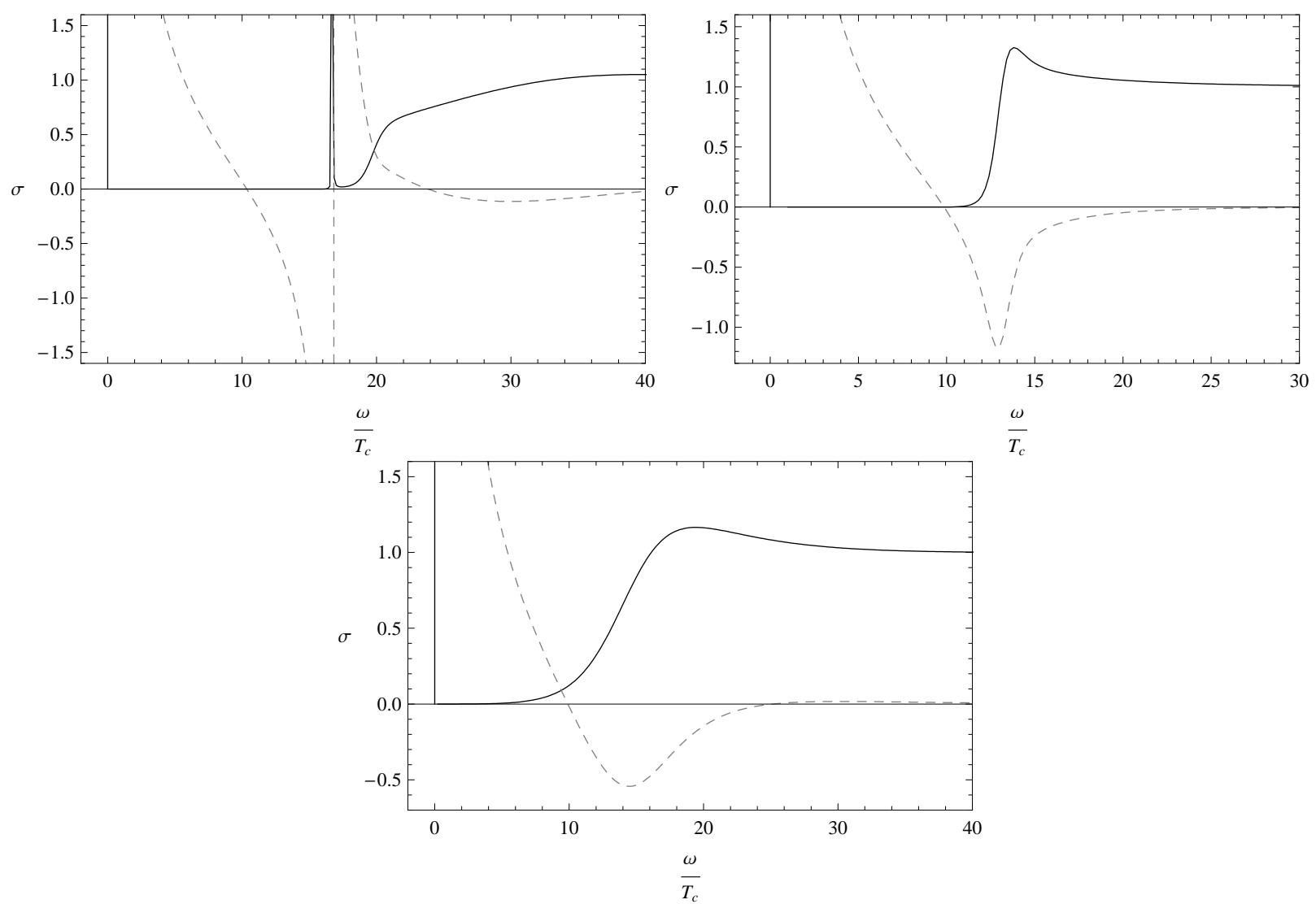

FIG. 3: The conductivity for operator $\left\langle\mathcal{O}_{1}\right\rangle$ at $T / T_{c} \approx 0.160$ (up-left); operator $\left\langle\mathcal{O}_{2}\right\rangle$ at $T / T_{c} \approx$ 0.205 (up-right) and operator $\left\langle\mathcal{O}_{3}\right\rangle$ at $T / T_{c} \approx 0.205$ (bottom). The solid curves denote the real part of conductivity $\operatorname{Re}[\sigma]$, while the dashed curves the imaginary part of conductivity $\operatorname{Im}[\sigma]$. For three cases the real parts all have a delta function at $\omega=0$, which means the infinite DC conductivity for superconductors.

frequency approaches zero. This phenomenon can be explained using the Kramers-Kronig relation

$$
\operatorname{Im}[\sigma(\omega)]=-P \int_{-\infty}^{\infty} \frac{d \omega^{\prime}}{\pi} \frac{\operatorname{Re}\left[\sigma\left(\omega^{\prime}\right)\right]}{\omega^{\prime}-\omega},
$$

where $P$ denotes the principal value of the integration. Because the real part has a delta function, $\operatorname{Re}[\sigma(\omega)] \sim \delta(\omega)$, we can get from (31) that $\operatorname{Im}[\sigma(\omega)] \sim \frac{1}{\pi \omega}$, which means there exists a pole at $\omega=0$.

We notice from the up-left plot of Fig. 3 that there is a spike in the conductivity, which also appears for the Schwarzschild-AdS black hole case in [32]. We will give a qualitative interpretation for this spike later. Despite of the spike, $\operatorname{Re}[\sigma(\omega)]$ exponentially decreases when $\omega \ll \omega_{g}$. Following the analysis in [32], for $\lambda>\lambda_{\mathrm{BF}}=\frac{3}{2}$, we define $\omega_{g}$ as the frequency which minimizes the imaginary part of conductivity $\operatorname{Im}[\sigma]$. So for cases with $\lambda>\lambda_{\mathrm{BF}}$ and $T / T_{c} \approx 0.205$, we find a roughly uniform ratio in Fig. 3 , that is,

$$
\frac{\omega_{g}}{T_{c}} \approx 13
$$


with the accuracy more than $93 \%$. This ratio is a little larger than the one in [32], but still in the same order. In the BCS theory, the energy to break apart the condensate is about $3.54 T_{c}$ when $T=0$, our result $\omega_{g} \approx 13 T_{c}$ indicates that in the Horava-Lifshitz superconductor the energy is larger than the one in the weakly coupled case.

It was also expected that when $\omega \ll \omega_{g}$, one has

$$
\operatorname{Re}[\sigma] \sim e^{-\Delta / T},
$$

where $\Delta$ is a constant. This implies that when $T \sim 0, \operatorname{Re}[\sigma]$ should be strictly zero. However, as stressed in [22, 23], $\operatorname{Re}[\sigma(\omega)]$ in fact depends on the frequency as a power law form, e.g., $\operatorname{Re}[\sigma(\omega)] \propto \omega^{\delta}$ with $\delta$ a function of some potential. Exploring the zero temperature limit of this Hořava-Lifshitz superconductor would be an interesting issue.

We can also see from Fig. 3 that when frequency is around $\omega_{g}, \operatorname{Re}[\sigma(\omega)]$ in the up-left plot grows slower than that in the up-right one. This different growing type may be expected from type II and type I coherence factors [7]. Because type II coherence will suppress absorption near $\omega_{g}$, the real part of conductivity will grow slower as the figure shows.

\section{B. Discussions on the Spike}

Here we try to give a qualitative explanation for the spike appearing in the up-left plot of Fig. 3. Following [22], we introduce a new radial variable $z$ as

$$
d z=\frac{d r}{f},
$$

It is easily seen that when $r \rightarrow \infty, f \sim r^{2}$, therefore, $z=-\frac{1}{r}$ at the boundary. At the horizon, $f \rightarrow 0$, therefore, $z=-\infty$ is the black hole horizon in the $z$ coordinate. Using (34), we can rewrite Eq. (27) as

$$
\text { - } A_{x, z z}+V(z) A_{x}=\omega^{2} A_{x} .
$$

with

$$
V(z)=2 q^{2} \psi^{2} f
$$

Eq. (35) is a one-dimensional Schrödinger equation. Then following [22], the conductivity can be expressed as

$$
\sigma(\omega)=\frac{1-\mathcal{R}}{1+\mathcal{R}},
$$

where $\mathcal{R}$ is the reflection coefficient. Naively we can deduce that if the barrier (potential $V(z)$ ) is very high and the incident energy of the incoming wave is lower than $V_{\max }, \mathcal{R}$ will be close to 1 , which means $\sigma(\omega)$ is very close to zero. From this analysis, $V_{\max } \approx \omega_{g}^{2}$. On the contrary, if $\omega^{2}>V_{\max }$, the incoming wave will easily cross over the barrier, therefore, $\mathcal{R} \rightarrow 0$ and $\sigma(\omega) \rightarrow 1$, which represents a normal state. The analysis above is consistent with what shows in Fig. 3 . 
In the case of $\lambda=1$, the potential at the boundary is

$$
V(z)=2 q^{2} \psi^{2} f \approx 2 q^{2}\left(\psi^{(1)}\right)^{2}
$$

where $\psi^{(1)}$ is a finite constant. At the horizon, $f \rightarrow 0$ and $\psi$ is finite, so $V(z) \rightarrow 0$ as $z \rightarrow-\infty$. Because $V(z)>0$ and it is continuous, we can deduce that $V(z)$ is bounded from above, i.e., $V(z) \leq V_{\max }$. The potential can be plotted as Fig.4. As is explained in [22],

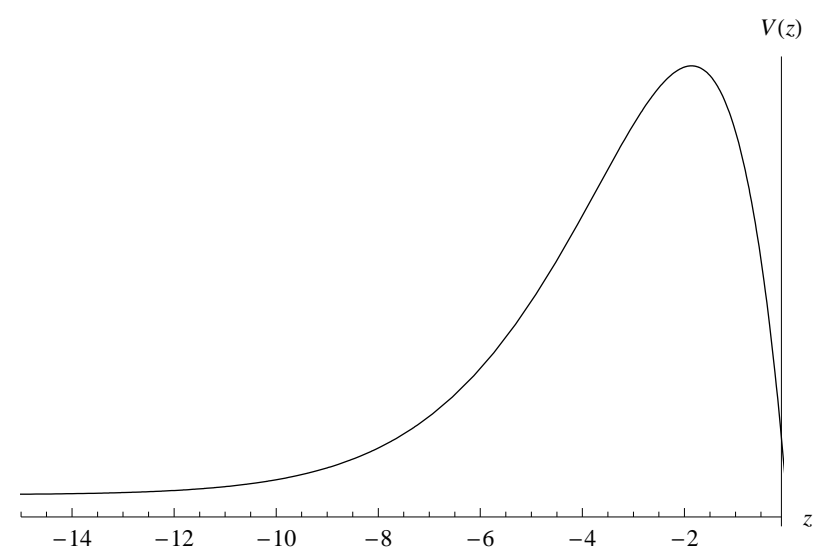

FIG. 4: The potential $V(z)$ which is bounded from above and goes to zero at the horizon $z \rightarrow-\infty$.

at low frequency the incoming wave is almost entirely reflected. But if one low frequency wavelength fits between the potential and the axis $z=0$, the reflected wave can interfere destructively with the incoming wave and cause the amplitude at $z=0$ to be exponentially small. According to the relation (37), this then produces a spike in $\sigma(\omega)$. It is suggested in [22] that by WKB approximation, there exists $\omega$ satisfying

$$
\int_{z_{0}}^{0} \sqrt{\omega^{2}-V(z)} d z+\frac{\pi}{4}=n \pi
$$

for some integer $n$, and $V\left(z_{0}\right)=\omega^{2}$, the spikes will appear. In our case, however, the potential $V(z)$ in the whole range of $z$ is difficult to have an analytic expression so that one is not able to analytically fix the position of the spike.

\section{CONCLUDING REMARKS}

In this paper we have studied the phase transition of planar black holes in the HořavaLifshitz theory by introducing a Maxwell field and a complex scalar field in the probe approximation. In particular, we have discussed the cases of $m^{2} L^{2}=-2$ and $m^{2}=0$, respectively. The former case shows that the two fallings of the complex scalar field are both normalizable modes. They are of scaling dimension one and two respectively. The later case has only a normalizable mode and the dual operator is of scaling dimension three. We found that the charged operator condensates behaving as $\left(T_{c}-T\right)^{1 / 2}$, near the critical temperature, which is qualitatively consistent with the BCS theory. In particular, the condensates increase 
as the scaling dimension gets larger. We computed the electrical conductivities in the dual CFT when the temperature is much below the critical temperature. The dual CFT will turn to a normal state when the frequency is higher than the critical frequency $\omega_{g}$. On the contrary, when the frequency is lower than $\omega_{g}$, the real part of the conductivity will decrease exponentially. There is an infinity DC conductivity at the zero frequency. In addition, it was found that when the scaling dimension of the charged operator is one, there exists a spike in the real part of the conductivity. We gave a qualitative explanation for this phenomenon using a one-dimensional Schrödinger equation. We found that our results are quite similar to those in the case of Schwarzschild-AdS black holes. It implies that the holographic superconductivity observed in [6, 7] is a robust phenomenon associated with asymptotic AdS black holes.

\section{Acknowledgments}

RGC would like to thank Kinki University, Osaka for warm hospitality during his visit. HQZ would like to thank T. Chern, B. Hu, Z.Y. Nie, S. Pu and Y. Zhou for helpful discussions. This work is supported partially by grants from NSFC, China (No. 10535060, No. 10821504 and No. 10975168) and a grant from MSTC, China (No. 2010CB833004).

[1] J. Maldacena, The Large N Limit of Superconformal Field Theories and Supergravity, Adv. Theor. Math. Phys. 2, 231 (1998). hep-th/9711200

[2] S.S. Gubser, I.R. Klebanov and A.M. Polyakov, Gauge Theory Correlators from Noncritical String Theory, Phys. Lett.B 428,105 (1998). hep-th/9802109

[3] E. Witten, Anti-de Sitter Space and Holography, Adv. Theor. Math. Phys. 2, 253 (1998). hep-th/9802150

[4] S.A. Hartnoll, Lectures on Holographic Methods for Condensed Matter Physics. arXiv:0903.3246,

[5] C.P. Herzog, Lectures on Holographic Superfluidity and Superconductivity. [arXiv: 0904.1975]

[6] S.S. Gubser, Breaking an Abelian Gauge Symmetry Near a Black Hole Horizon, Phys. Rev. D 78, 065034 (2008). arXiv:0801.2977.

[7] S.A. Hartnoll, C.P. Herzog and G.T. Horowitz, Building an AdS/CFT superconductor, Phys. Rev. Lett. 101, 031601 (2008). arXiv:0803.3295]

[8] P. Basu, A. Mukherjee and H.H. Shieh, Supercurrent: Vector Hair for an AdS Black Hole, Phys. Rev. D 79,126004(2009). arXiv:0809.4494

[9] C. P. Herzog, P. K. Kovtun and D. T. Son, Holographic model of superfluidity, Phys. Rev. D 79,066002 (2009). arXiv:0809.4870

[10] S.S. Gubser, Phase Transitions Near Black Hole Horizons, Class.Quant.Grav. 22,5121 (2005). hep-th/0505189

[11] S. Weinberg, Superconductivity for Particular Theorists, Prog. Theor. Phys. Suppl. 86, 43 (1986).

[12] R. D. Parks, Superconductivity, Marcel Dekker Inc. (1969). 
[13] S. A. Hartnoll, C. P. Herzog and G. T. Horowitz, Holographic Superconductors, JHEP0812:015,2008. arXiv:0810.1563.

[14] S. S. Gubser. Colorful horizons with charge in anti-de Sitter space. Phys. Rev. Lett.101,191601(2008). arXiv:0803.3483]

[15] M. M. Roberts and S. A. Hartnoll, Pseudogap and time reversal breaking in a holographic superconductor, JHEP 08:035, 2008. arXiv:0805.3898

[16] S. S. Gubser and S. S. Pufu, The gravity dual of a p-wave superconductor, JHEP 11:033, 2008. arXiv:0805.2960]

[17] F. Denef and S. A. Hartnoll. Landscape of superconducting membranes. arXiv:0901.1160]

[18] S.S. Gubser, C. P. Herzog, S.S. Pufu and T. Tesileanu, Superconductors from Superstrings, Phys. Rev. Lett. 103, 141601 (2009). arXiv:0907.3510

[19] J.P. Gauntlett, J. Sonner and T. Wiseman, Holographic superconductivity in M-Theory, Phys. Rev. Lett. 103,151601 (2009). arXiv:0907.3796

[20] S.S. Gubser, S.S.Pufu and F.D. Rocha, Quantum critical superconductors in string theory and M-theory. arXiv:0908.0011

[21] S.S. Gubser and A. Nellore, Ground states of holographic superconductors. arXiv:0908.1972

[22] G.T. Horowitz and M.M. Roberts, Zero Temperature Limit of Holographic Superconductors. arXiv:0908.3677.

[23] R.A. Konoplya and A. Zhidenko, Holographic conductivity of zero temperature superconductors, [arXiv: 0909.2138]

[24] E.J. Brynjolfsson, U.H. Danielsson, L. Thorlacius and T. Zingg, Holographic Superconductors with Lifshitz Scaling. arXiv:0908.2611.

[25] Sang-Jin Sin, Shan-Shan Xu and Yang Zhou, Holographic Superconductor for a Lifshitz Fixed Point. arXiv:0909.4857

[26] S. Cremonesi, D. Melnikov and Y. Oz, "Stability of Asymptotically Schroedinger RN Black Hole and Superconductivity, arXiv:0911.3806 [hep-th].

[27] P. Hořava, Quantum Gravity at a Lifshitz Point,Phys. Rev. D 79, 084008(2009). arXiv:0901.3775,

[28] Rong-Gen Cai, Li-Ming Cao and Nobuyoshi Ohta, Topological Black Holes in Horava-Lifshitz Gravity, Phys. Rev. D 80, 024003 (2009). arXiv:0904.3670.

[29] P. Breitenlohner and D.Z. Freedman, Stability in Gauged Extended Supergravity, Annals of Physics 144, 249 (1982).

[30] I.R. Klebanov and E. Witten, AdS/CFT Correspondence and Symmetry Breaking, Nucl.Phys.B 556, 89 (1999). hep-th/9905104

[31] M. Tinkham, Introduction to Superconductivity, 2nd e dition, Dover: New York (1996).

[32] G.T. Horowitz and M.M. Roberts, Holographic superconductors with various condensates, Phys. Rev. D78, 126008(2008).[arXiv: 0810.1077] 